\title{
Low radiation level detection with room temperature InAs detector
}

\author{
MAKAI Janos ${ }^{1}$, MAKAI Tamas ${ }^{2}$ \\ ${ }^{1}$ Hungarian Academy of Sciences, Research Center for Natural Sciences, Institute of \\ Technical Physics and Material Science, Budapest, Hungary \\ ${ }^{2}$ Graz University of Technology, Institute for Optimisation und Discrete Mathematics, Graz, \\ Austria
}

\begin{abstract}
Photovoltaic detectors are widely used in radiometry, photometry, spectroscopy, radiation thermometry, etc. from the UV to the IR wavelength range. As the wavelength to be measured increases from the UV to IR, different semiconductors are to be used, and with increasing wavelength the band-gap and the shunt resistance of the $\mathrm{p}-\mathrm{n}$ junction decreases. Typical values at room temperature are: $\mathrm{GaP}$ bandgap $=2,25 \mathrm{eV}$ shunt resistance $=10^{12} \Omega$ peak responsivity $=0,44 \mu \mathrm{m}, \mathrm{Si}$ bandgap $=1,11 \mathrm{eV}$ shunt resistance $=10^{9}-10^{10} \Omega$ peak responsivity $=0,85 \mu \mathrm{m}$, InGaAs bandgap $=0,75 \mathrm{eV}$ shunt resistance $=10^{8}-10^{9} \Omega$ peak responsivity $=1,5 \mu \mathrm{m}, \mathrm{Ge}$ bandgap $=0,66 \mathrm{eV}$ shunt resistance $=10-100 \mathrm{M} \Omega$ peak responsivity $=1,7 \mu \mathrm{m}$, InAs bandgap $=0,36 \mathrm{eV}$ shunt resistance $=10-100 \Omega$ peak responsivity $=3,1 \mu \mathrm{m}$.
\end{abstract}

To achieve the highest linearity photovoltaic detectors are used in the zero bias mode, i.e. they are connected to a current-to-voltage (I/V) converter. Ideally, the input impedance of the I/V converter is negligible compared to the shunt impedance of the detector, consequently the whole photogenerated current flows on the feedback impedance and will produce the output voltage, otherwise a part of it flows on the shunt impedance. A current on the shunt impedance causes a deviation from the zero bias mode, it changes the electric field in the $\mathrm{p}-\mathrm{n}$ junction i.e. affecting recombination rate and collection factor of the generated electron-hole pairs. In most cases, upto the InGaAs detector the input impedance of the I/V converter at low frequencies is negligible compared to that of the detector. The situation with the low band-gap InAs detector is perfectly different, the impedances even can be in the same order of magnitude. Since low level detection requires high feedback resistance, at sufficiently high feedback impedance value the output signal shall deviate from the ideal case, in the worst case the whole photogenerated current can flow on the shunt impedance generating close to zero output voltage. Further, the shunt impedance changes with temperature and the input impedance with the change of range, the latter one is linearly proportional to the value of the feedback resistor, consequently the ratio of the input impedance to shunt impedance changes affecting the linearity.

The linearity of $\mathrm{Si}$, InGaAs and Ge detectors connected to an $\mathrm{I} / \mathrm{V}$ converter are thoroughly discussed in the literature, contrary to InAs detector. In an earlier work ${ }^{1}$ it has been shown that applying a bootstrap circuit to a Ge detector - depending on the frequency of the operation - will virtually increase by 3-6 decades the shunt impedance of the detector compared to the detector alone. In the present work a similar circuitry was applied to a room temperature InAs detector. It will be shown, how the bootstrap circuit channels the photogenerated current to the feedback impedance decreasing with many decades the detectable low level limit of the detector I/V converter unit. The linearity improvement results will be shown as a function of the chopping frequency, calculated and measured values will be compared, the noise sources will be analyzed and noise measurement results will be presented.

Keywords: radiometry, photometry, detector, linearity, photovoltaic, bootstrap

\section{INTRODUCTION}

For high accuracy radiometric measurements a detector is required that together with the attached circuit provides a linear response signal over the whole radiative power range to be measured. Recently, room temperature or near room temperature InAs detectors are widely used in laser warning receivers, process control

\footnotetext{
${ }^{1}$ Corresponding author: makai.janos@ttk.mta.hu
} 
monitors, temperature sensors, pulsed laser monitors, infrared spectroscopy, etc. ${ }^{2}$. If calibration takes place, detectors are calibrated against cryogenic radiometers at a signal level of a couple of $100 \mu \mathrm{W}$, but for low level radiation measurements they are used sometimes at a signal level of 6-8 decades lower. In some applications like in high accuracy spectrophotometry where the ratio of two measurement results provide information on a physical property as of transmittance, reflectance or absorbance, the two signals can be many decades apart. Temperature measurement should provide a linear scale over a large range. Many of the applications impose huge demand on the linearity of the measurement system. In our investigations all the measurements and calculations were carried out for a room temperature InAs p-n photodiode, but our results can be used at any low shunt resistance photodiode. In this paper the linearity of low shunt resistance photovoltaic detectors (PV) together with the attached electronic circuit will be discussed, the main emphasis will be given to the interaction of the detector and the attached circuit.

A photovoltaic detector is a p-n or p-i-n junction with a depletion region having a high electric field. This field serves for the separation of the by the incident radiation generated electron-hole pairs ${ }^{3}$. Pairs can be generated either in the depletion region or in a region where a carrier can diffuse to the depletion region with some probability. Photovoltaic detectors are usually attached to current - to - voltage (I/V) converter that should provide a stable condition (zero bias) for the detector at any range. The zero bias ensures that the electric field in the depletion region will remain constant independently from the amount of the photogenerated current, consequently the probability of the collection will be the same at any circumstances. The I/V converter under proper conditions provides a virtual ground for the detector, the inherent electric field is not effected by the detection electronics. Unfortunately, the zero bias is kept only until the feedback impedance is not much larger, then that of the photodiode. For detectors of higher bandgap and shunt resistance ( $\mathrm{GaP}, \mathrm{Si}, \mathrm{InGaAs})$ this condition is met at low frequencies. The situation with low or lower bandgap detectors (Ge, InAs, InSb) is totally different. It means that, when the photodiode is irradiated a bias appears on the photodiode, it superimposes on the electric field of the depletion region influencing the separation - collection efficiency and by that a change in the dark current appears. It has been shown, that the dark current originates from injection diffusion and generation - recombination through surface shunt, bulk leakage and tunneling at the surface that depends on the passivation of the surface in an InAs $p-n$ junction, as well as from background radiation. The dark current and the shunt resistance of the InAs photodiode strongly depends on the temperature. ${ }^{4}$ Consequently, any small change in the internal impedance of the detector due to temperature change or its ratio to the measuring circuit will affect the measured signal. Leakage and tunneling can be decreased by cooling the detector, but the background radiation remains. For mobile equipment the cooling is also non practical. Even if high bandgap detectors are used at very high sensitivity measurements, that is very high feedback resistance is applied compared to the internal resistance of the detector, this degenerating phenomenon will be experienced.

\section{CURRENT - TO - VOLTAGE (I/V) CONVERTER}

A current - to - voltage (I/V) converter is shown on Fig. 1. $\mathrm{Ph}$ is the photodiode, $\mathrm{OP}_{1}$ is the operational amplifier, $Z_{\mathrm{f}}$ is the feedback impedance and $U_{\text {out }}$ is the output voltage of the $\mathrm{I} / \mathrm{V}$ converter where ideally $U_{\text {out }}=I_{\mathrm{p}} * Z_{\mathrm{f}}$ and $I_{\mathrm{p}}$ is the photogenerated current.

The I/V converter theoretically ensures stable electrical conditions for the photodiode independently from the incident radiative power. In the depletion region collected carriers are swept out by the built in electric field to the feedback resistor. Because the I/V converter under proper conditions provides a

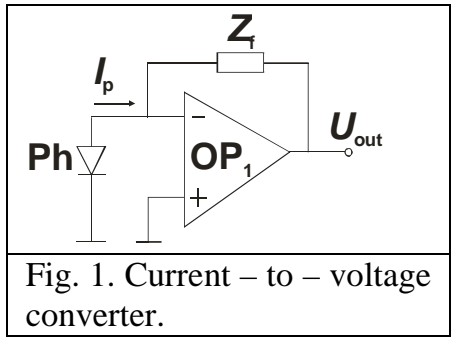
virtual ground for the detector the inherent electric field is not effected by the detection electronics.

Fig. 2. shows a simplified equivalent circuit of the I/V converter together with the photodiode. $Z_{\mathrm{d}}$ is a nonlinear impedance representing the diode, the so called shunt impedance, $Z_{\mathrm{s}}$ is the series resistant and $Z_{\text {in }}$ is the input impedance of the $\mathrm{I} / \mathrm{V}$ converter. For high shunt resistance detectors $(\mathrm{GaP}, \mathrm{Si})$, when $Z_{\mathrm{d}} \gg Z_{\mathrm{s}}, Z_{\mathrm{s}}$ can be neglected, but for InAs detectors they are in the same magnitude. The shunt resistance of a photovoltaic detector is defined at DC signals and zero bias and it is the first derivative of the dark current

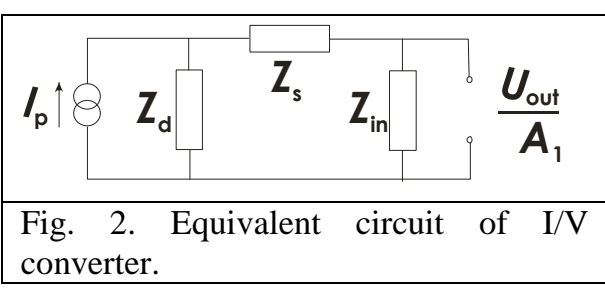
with respect to the applied voltage between the poles of the detector. The shunt impedance can be defined similarly but with AC signals; consequently, it has a frequency dependency. The shunt impedance/resistance of the photodetector has a strong temperature dependency as shown in ${ }^{4,5}$. The output voltage is transformed to the input impedance of the $\mathrm{I} / \mathrm{V}$ converter. The transformation factor is $A_{1}$, in the ideal case the open loop gain of the 
operational amplifier. $Z_{\text {in }}=Z_{\mathrm{f}} /\left(A_{1}+1\right)$. If $Z_{\text {in }}<<Z_{\mathrm{d}}$ then the photogenerated current flows through $Z_{\text {in }}$ otherwise a part of it appears on $Z_{\mathrm{d}}$ and will not produce output voltage. This equivalent circuit is strictly true only for the ideal case, for non-ideal one it does not describe the physical phenomenon, but as it will be seen it provides a satisfactory model for the comprehension of the final result. If $\left(Z_{\mathrm{s}}+Z_{\text {in }}\right)<<Z_{\mathrm{d}}$ then the output voltage corresponds to the whole photogenerated current. Either $Z_{\mathrm{s}}$ or $Z_{\text {in }}$ will increase so far that the previous inequality will not hold, it will degenerate the electric field in the depletion region. On the other hand, as the frequency increases $A_{1}$ decreases i.e. both the zero bias of the photodiode and the constant electric field of the p-n junction degenerate.

Fig. 3. shows a typical curve of open-loop gain $\left(A_{1}\right)$ of an operational amplifier as a function of frequency. It can be seen that at DC its value is $120 \mathrm{~dB}$ and from a couple of $\mathrm{Hz}$ on it decreases with $20 \mathrm{~dB} / \mathrm{decade}$. The output voltage of the $\mathrm{I} / \mathrm{V}$ converter is influenced by the closed loop gain $(H)$, too. $H=A_{1} * \beta$ where $\beta$ is the feedback factor i.e. $\beta=\left(Z_{\mathrm{d}}+Z_{\mathrm{s}}\right) /\left(Z_{\mathrm{d}}+Z_{\mathrm{s}}+Z_{\mathrm{f}}\right)$. The higher the closed loop gain is the less the amplitude error $\left(A_{\mathrm{err}}\right)$ of the output of the I/V converter is and vice versa. $H$ and $1 / \beta$ were marked on Fig. 3. From Fig. 3. can be seen as $1 / \beta$ increases, i.e. for example $Z_{\mathrm{f}}$ increases compared to $Z_{\mathrm{d}}$ then $H$ decreases and $A_{\text {err }}$ increases. $A_{\mathrm{err}}=1 /(1+H)$. Consequently, $A_{\text {err }}=\left(Z_{\mathrm{d}}+Z_{\mathrm{s}}+Z_{\mathrm{f}}\right) /\left[\left(A_{1}+1\right) *\left(Z_{\mathrm{d}}+Z_{\mathrm{s}}\right)+Z_{\mathrm{f}}\right]$ This equation shows, that either $Z_{\mathrm{d}}$ should be larger than $Z_{\mathrm{s}}+Z_{\mathrm{f}}$, or if $Z_{\mathrm{d}}<\left(Z_{\mathrm{s}}+Z_{\mathrm{f}}\right)$, $\left(A_{1}+1\right)^{*} Z_{\mathrm{d}}$ should be much higher than $\left(A_{1}+1\right)^{*} Z_{\mathrm{s}}+Z_{\mathrm{f}}$. Unfortunately, $A_{1}$ decreases with increasing frequency even in the low frequency range. Since IR photodetectors generally measure chopped radiation to exclude the effect of the background radiation and that of the dark current this worsens the situation compared to DC detection. If the temperature of a non temperature stabilized photodiode changes then $Z_{\mathrm{d}}$ and with it $A_{\text {err }}$ changes. For

OPEN LOOP GAIN OF AN OPERATIONAL AMPLIFIER

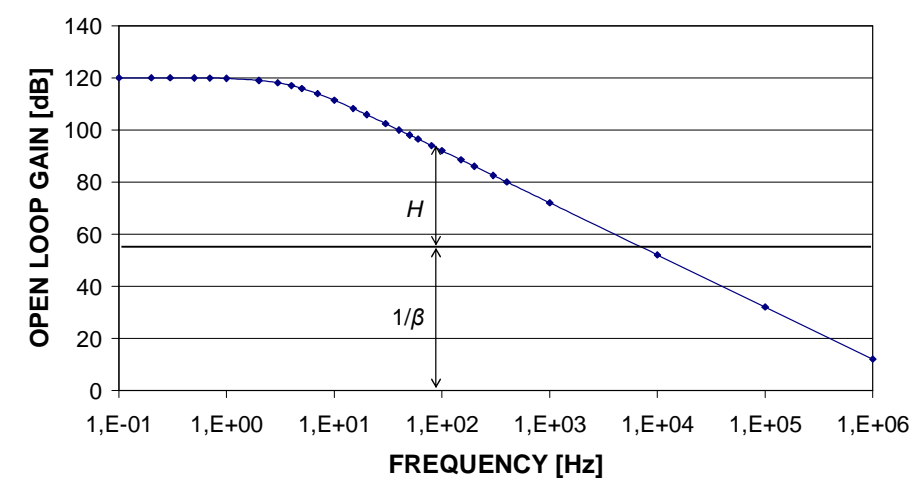

Fig. 3. Open loop gain of an operational amplifier. example the temperature coefficient of $Z_{\mathrm{d}}$ is in the $10 \% / \mathrm{K}^{\circ}$ range for a Ge detector ${ }^{5}$.

\section{BOOTSTRAPPING OF THE PHOTOVOLTAIC DETECTOR}

To keep the closed loop gain at a high value or to inhibit its change can be achieved only if the impedance of the photodiode is virtually increased, it means the voltage on the photodiode is kept constant, preferably at a low level, i.e. the photodiode is bootstrapped. Fig 4. shows the schematic of the circuit. This schematic circuit was originally devised by Campastro ${ }^{6}$ for high frequency applications to shunt the capacitance of the diode. $\mathrm{OP}_{1}$ is the $\mathrm{I} / \mathrm{V}$ converter and $\mathrm{OP}_{2}$ is a voltage follower. The voltage follower ensures the almost zero bias condition, on the photodiode appears only the differential input voltage of $\mathrm{OP}_{2}$ independently from the state of $\mathrm{OP}_{1}$. This solution

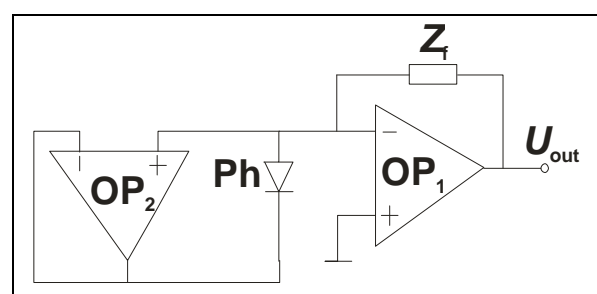

Fig. 4. Bootstrap circuit for a photodiode. virtually increases the impedance of the photodiode by a factor of $A_{2}$, the open loop gain of the follower ${ }^{7}$ producing a high closed loop gain for the I/V converter. Fig. 5. shows the equivalent circuit of the bootstrapped photodiode connected to an I/V converter. It was assumed that the offset voltage of the bootstrap amplifier was set to zero. Even if it is not zeroed, only a constant offset voltage will appear on the photodiode, i.e. the electric field in the $p-n$ junction remains constant independently from the photocurrent and the value of the feedback impedance. As from the equivalent circuit could be seen, the equations given in the previous section should be modified, in all of them $Z_{\mathrm{d}}$ and $Z_{\mathrm{s}}$ should be replaced by $A_{2} * Z_{\mathrm{d}}$ and $A_{2} * Z_{\mathrm{s}}$, respectively. The formula for the amplitude error then becomes $A_{\text {err }}=\left[A_{2} *\left(Z_{\mathrm{d}}+Z_{\mathrm{s}}\right)+Z_{\mathrm{f}}\right] /\left[\left(A_{1}+1\right) * A_{2} *\left(Z_{\mathrm{d}}+Z_{\mathrm{s}}\right)+Z_{\mathrm{f}}\right]$. Using a similar reasoning $A_{2} * Z_{\mathrm{d}}$ should be larger than $A_{2} * Z_{\mathrm{s}}+Z_{\mathrm{f}}$ or if $A_{2} * Z_{\mathrm{d}}<\left(A_{2} * Z_{\mathrm{s}}+Z_{\mathrm{f}}\right)$ then $A_{2} *\left(A_{1}+1\right) * Z_{\mathrm{d}}$ should be much higher

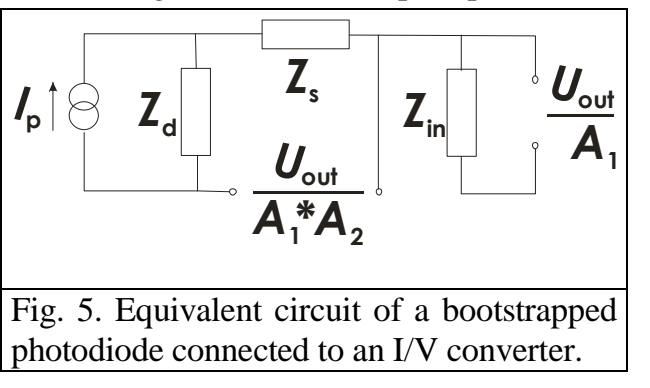
than $A_{2} *\left(A_{1}+1\right) * Z_{\mathrm{S}}+Z_{\mathrm{f}}$. to get a low amplitude error. The deviation described in the previous section for the $\mathrm{I} / \mathrm{V}$ converter appears only at a much higher feedback impedance value, allowing, depending on the chopping 
frequency $3-5$ decade higher linear sensitivity. To have this system operated properly the slew rate of the voltage follower should be higher then that of the I/V converter. Contrary to the I/V converter circuit here not $A_{1}$ but the $A_{1} * A_{2}$ product should decrease to degenerate the zero bias condition.

\section{RESULTS}

To test the performance of this circuit a $2 \mathrm{~mm}$ diameter room temperature InAs detector was used. The feedback resistor was varied from $100 \mathrm{k} \Omega$ to $1 \mathrm{G} \Omega$ without any capacitance but the stray one and the frequency was varied from $10 \mathrm{~Hz}$ to $400 \mathrm{~Hz}$. The configuration of the circuitry could be changed from $\mathrm{I} / \mathrm{V}$ converter to bootstrapped photodiode - I/V converter by the help of a three circuit single switch. To get a stable radiation we used a tungsten halogen lamp with a monochromator, the set wavelength was $2560 \mathrm{~nm}$. All of the measurements were carried out at this wavelength, except the very high sensitivity ones, where to avoid the overload a higher wavelength was used. This was taken into account by a correction factor, similarly to the range changes. The radiation was chopped by a mechanical chopper that in ideal case resulted in a time dependent irradiation on the detector and therefore in a trapezoidal photogenerated signal. Fig. 6. shows the results in logarithmic scale. It could be seen, that for the simple I/V circuitry only the $100 \mathrm{k} \Omega$ feedback resistor provides an acceptable linearity throughout the whole frequency range, even the $1 \mathrm{M} \Omega$ feedback causes an $11 \%$ decrease from $10 \mathrm{~Hz}$ to $400 \mathrm{~Hz}$. We usually measure at $121 \mathrm{~Hz}$, to be as far as possible from the harmonics of the 50

MEASURED VALUES BY LOCK-IN AMPLIFIER USING I/V CONVERTER AND BOOTSTRAPPED PHOTODIODE - I/V CONVERTER CIRCUITRY

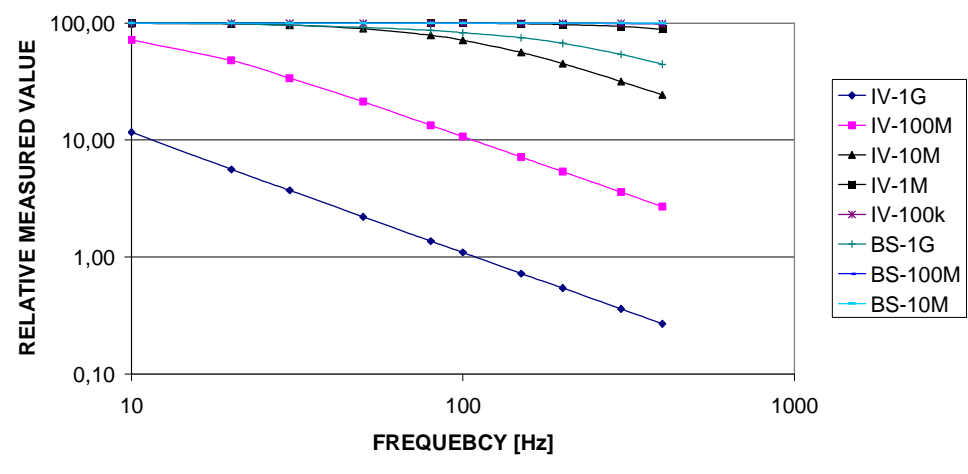

Fig. 6. Frequency dependent measured values of the same optical radiation using $\mathrm{I} / \mathrm{V}$ converter and bootsrapped photodiode - I/V converter circuitry.

$\mathrm{Hz}$ power, it means that one can use even the $1 \mathrm{M} \Omega$ feedback at this frequency with about a $1,3 \%$ decrease. On the other hand the bootstrap circuitry provides linear measurements upto the $100 \mathrm{M} \Omega$ feedback, it arrives to the $1 \%$ decrease with the $1 \mathrm{G} \Omega$ feedback only at $25 \mathrm{~Hz}$. For the clarity of the figure the curves of the $1 \mathrm{M} \Omega$ and 100 $\mathrm{k} \Omega$ feedbacks for the bootstrap variation were omitted, they just give a horizontal straight line over the $10 \mathrm{M} \Omega$ one.

Fig 7. and Fig. 8. show the signal responses of the traditional I/V converter and the bootstrapped variation, respectively, taken at $30 \mathrm{~Hz}$. The conditions under the curves of Fig. 7. and 8. were taken are the same. The curves were normalized and shifted to make the visualization easier. Note that from Fig. 6 . it is known, that the amplitude of these curves can be decades apart. Fig 8. confirmes that the bootstrap curve shows a near ideal operation of the circuit as an I/V converter, the qualitative improvement is evident. On the lowest sensitivity I/V converter curve and on all bootstrap curves higher frequency noise components are superimposed, showing the better frequency response of these cases. Essentially, this does not affect the measured values, since the lock-in amplifier measures only the amplitude of the base harmonics. For a triangular function the amplitude of the base harmonics is even lower, than for a trapezoidal function of the same amplitude, making the trapezoidal function more favorable. 


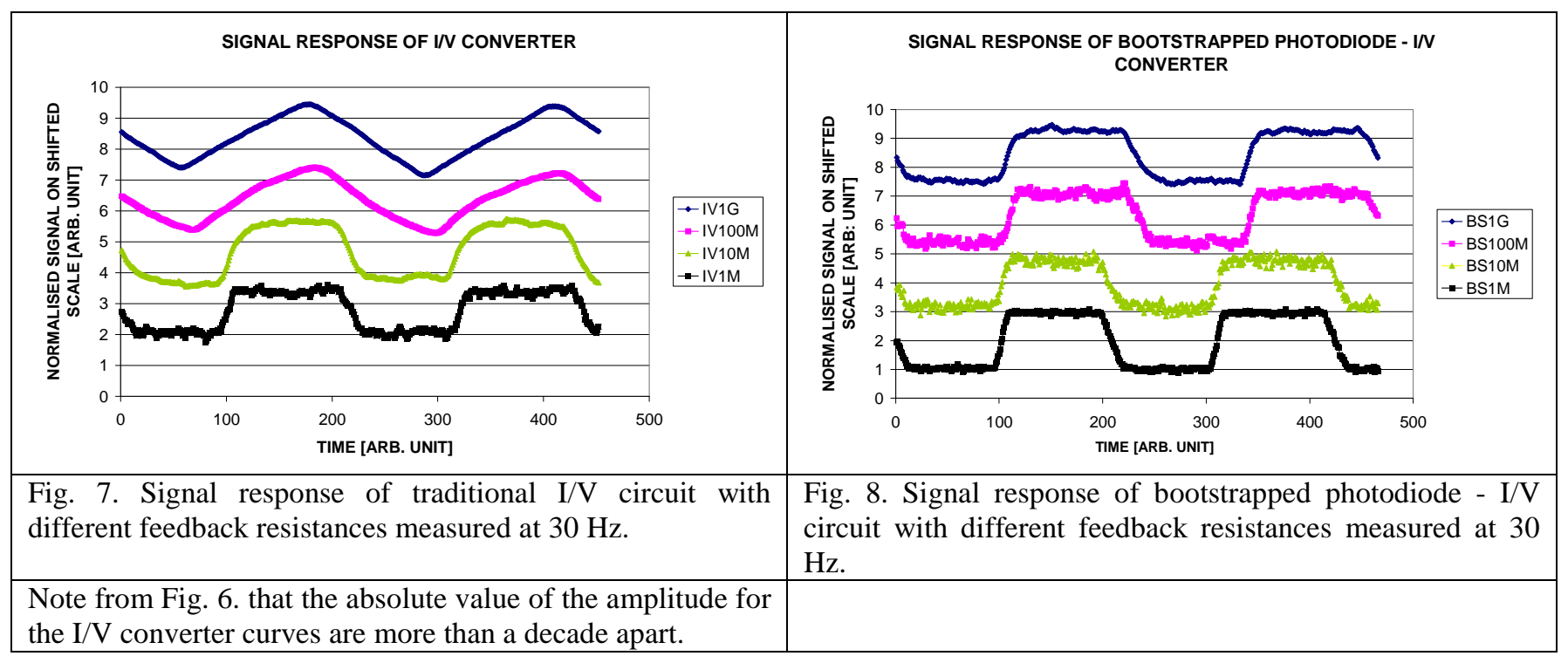

Fig. 9. shows the change of the time response at $10 \mathrm{M} \Omega$ feedback resistor for the simple $\mathrm{I} / \mathrm{V}$ converter as a function of the frequency. It can be seen, that from $10 \mathrm{~Hz}$ to $400 \mathrm{~Hz}$ how the shape of the curves changes from trapezoidal function to triangular function and at the same time the amplitude decreases to about 1/4th of its initial value. Since the frequency of good quality mechanical choppers changes a couple of percent during a long measurement series, this results also in a change of the measured signal.

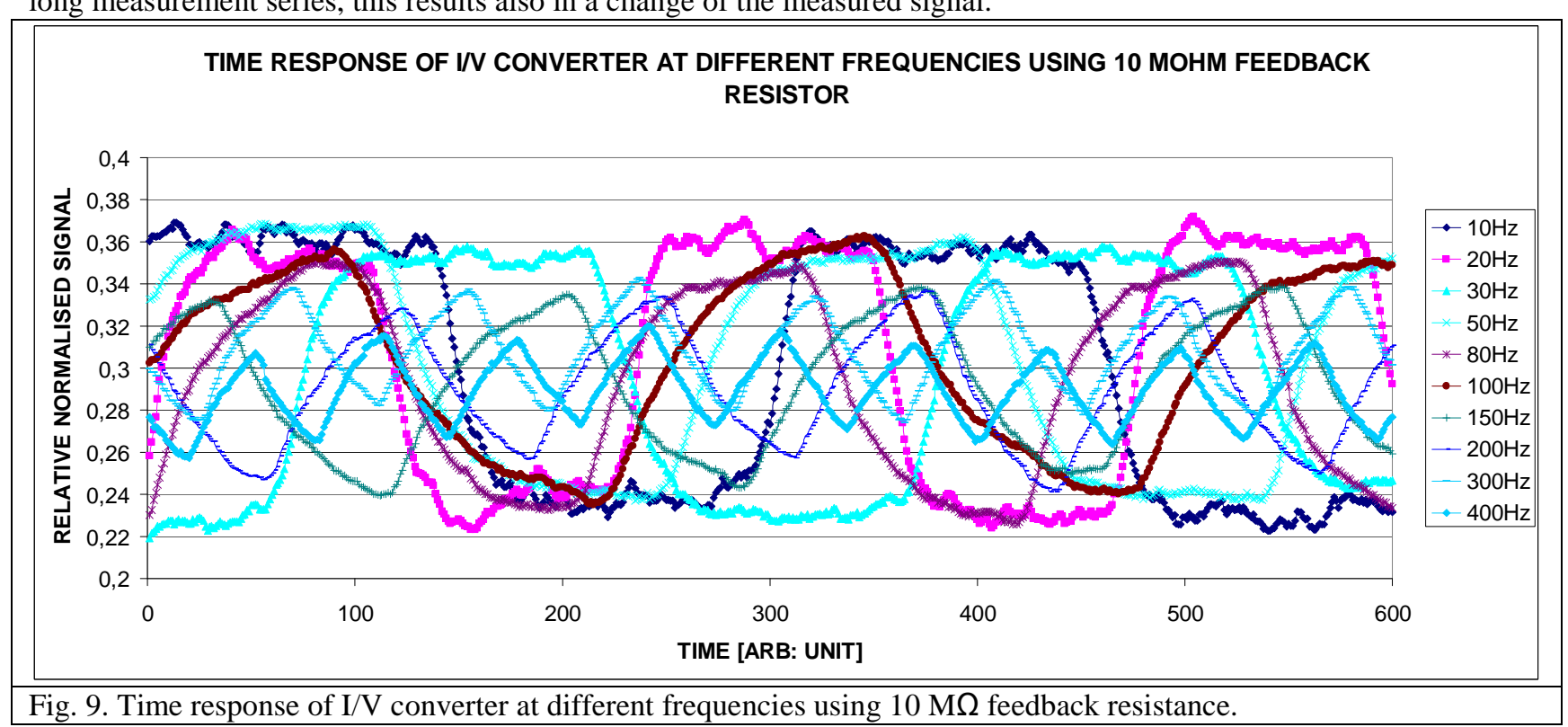

From the amplitude error $A_{\text {err }}$ formulae the signal amplitude can be calculated (1- $\left.A_{\text {err }}\right)$ and compared with the measured signals. Figs. 10. and 11. show these results for the simple I/V converter in logarithmic and the ratio of the measured to calculated signal in linear scale, respectively. The deviation of the calculated signal amplitude from the measured values results from the frequency dependent nonlinearity of the open-loop-gain, that also depends on the attached impedances, from the diode nonlinearity, the stray capacitances, lead inductances and measurement errors. It can be seen, that Fig. 10. provides very similar curves to sections of the open loop gain of an operational amplifier shown in Fig. 3. Since at low frequencies the feedback factor is constant, as the frequency increases the closed loop gain decreases and the amplitude error, the deviation from the ideal horizontal line, increases. For the calculations an ideal open-loop-gain was assumed. The open parameters used for a best fit solution were the shunt resistance of the photodiode, stray capacitance, and $3 \mathrm{~dB}$ point of the open-loop-gain. It resulted in a shunt resistance about $100 \Omega$, that is a usual value for an InAs room temperature photodiode and the deviation between the measured and calculated values were less then $\pm 20 \%$. In the legends $m$ indicates the measured and $\mathrm{c}$ the calculated values. Similar calculations were carried out for the bootstrapping variation, the error upto the $100 \mathrm{M} \Omega$ feedback resistor for all chopping frequencies was less than $1 \%$, for the $1 \mathrm{G} \Omega$ feedback it slightly exceeded the $10 \%$. 


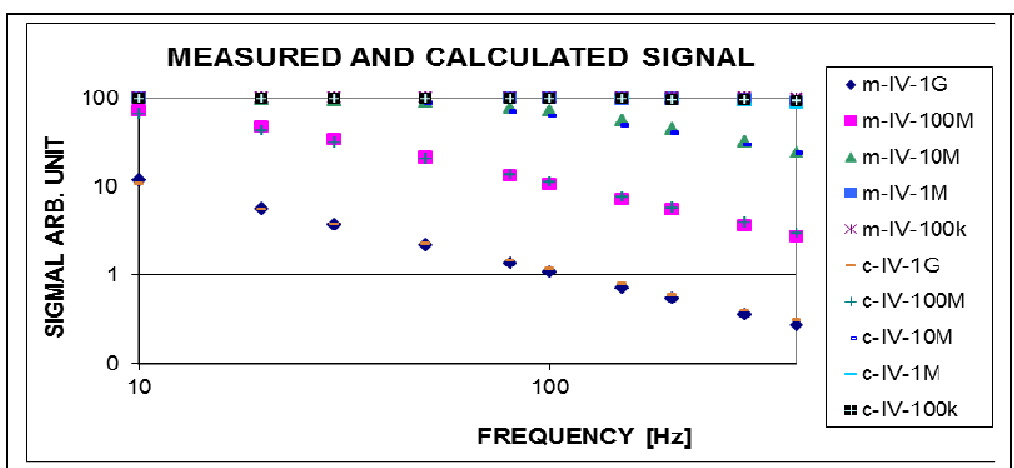

Fig. 10. Measured and calculated signal amplitudes for the InAs photodiode connected to an I/V converter as a function of frequency.

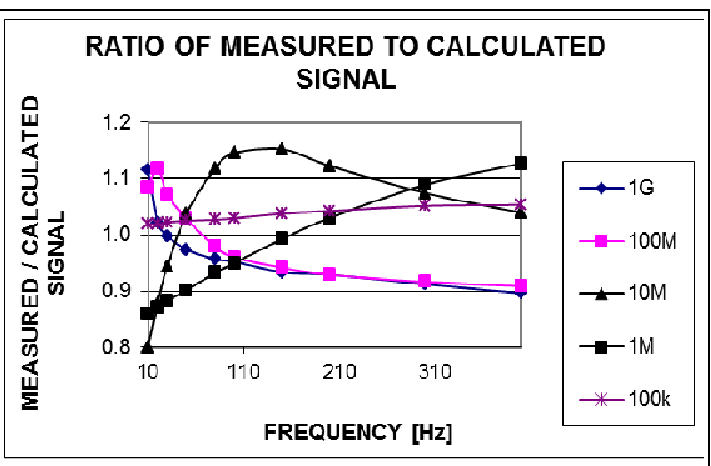

Fig. 11. Ratio of the measured to calculated signal as a function of frequency.

\section{NOISE ANALYSES}

Fig. 12. shows the noise equivalent circuit of the bootstrap variation. For simplicity only impedances are shown all of their real resistance components, if used later are referred to by the same index. The traditional current-tovoltage converter has noise sources from the detector, the amplifier and the feedback resistor. $U_{1 \mathrm{n}}$ and $U_{2 \mathrm{n}}$ represent the amplifier input voltage noise, $I_{1 \mathrm{n}}$ and $I_{2 \mathrm{n}}$ the input current noise, $I_{\mathrm{dn}}$ involves all the noises originating from the detector.

By applying the bootstrap the output noise originating from the feedback resistor, input noise current of the I/V converter and detector are not affected. The noise gain decreases for the input voltage noise of $\mathrm{OP}_{1}$ because the source impedance increased but the input voltage noise of $\mathrm{OP}_{2}$ is amplified with a gain of $Z_{\mathrm{f}} /\left(Z_{\mathrm{d}}+Z_{\mathrm{s}}\right)$. The input current noise of $\mathrm{OP}_{2}$ appears directly on $Z_{\mathrm{f}}$. Summing up the spectral noise components at the output:

for current-to-voltage converter:

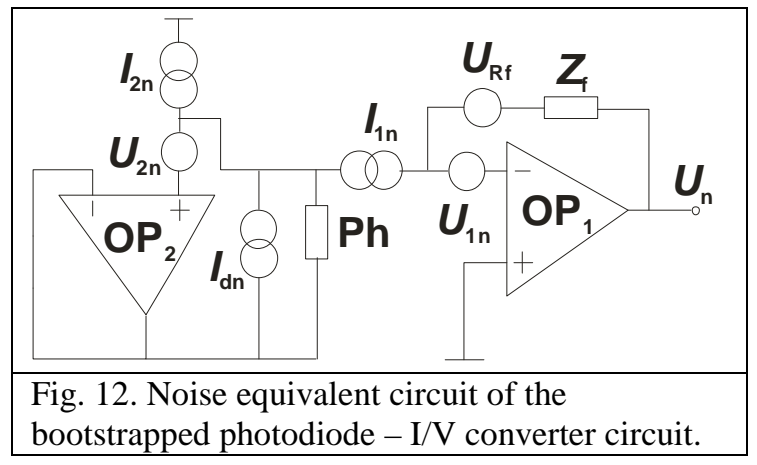

$U_{n}=\sqrt{\left(I_{d n} \cdot Z_{f}\right)^{2}+\left(I_{1 n} \cdot Z_{f}\right)^{2}+\left[\left(1+\frac{Z_{f}}{Z_{d}+Z_{s}}\right) \cdot U_{1 n}\right]^{2}+4 k T R_{f}}$

for current-to-voltage converter with bootstrapped photodiode:

$U_{n}=\sqrt{\left(I_{d n} \cdot Z_{f}\right)^{2}+\left(I_{1 n} \cdot Z_{f}\right)^{2}+\left[\left(1+\frac{Z_{f}}{\left(Z_{d}+Z_{s}\right)\left(A_{2}+1\right)}\right) \cdot U_{1 n}\right]^{2}+4 k T R_{f}+\left(I_{2 n} \cdot Z_{f}\right)^{2}+\left(\frac{Z_{f}}{Z_{d}+Z_{s}} U_{2 n}\right)^{2}}$

If equations 1 and 2 are compared, it can be seen that bootstrapping does not result in significant increase of the noise. This has been verified experimentally by measuring the spectral noise at $121 \mathrm{~Hz}$ with an SR830 lock-in amplifier. Table 1. shows the results for the $100 \mathrm{k} \Omega$ and $1 \mathrm{M} \Omega$ feedback resistors. At higher feedback resistor values the noise gain for the $\mathrm{I} / \mathrm{V}$ converter is already impaired by the non-ideal operation, consequently a comparison has no sense.

\begin{tabular}{|c|c|c|}
\hline Feedback resistor & I/V converter & Bootstrapped photodiode I/V converter circuit \\
\hline $100 \mathrm{k} \Omega$ & $45,67 \mu \mathrm{V} / \sqrt{\mathrm{Hz}}$ & $57,08 \mu \mathrm{V} / \sqrt{\mathrm{Hz}}$ \\
\hline $1 \mathrm{M} \Omega$ & $34,62 \mu \mathrm{V} / \sqrt{\mathrm{Hz}}$ & $35,22 \mu \mathrm{V} / \sqrt{\mathrm{Hz}}$ \\
\hline
\end{tabular}




\section{CONCLUSIONS}

Using an I/V converter with a photodetector, the ratio of the photodetector impedance to the quotient of the feedback impedance and the open loop gain of the amplifier; i.e. the input impedance of the I/V converter, can have a dominant role in the accuracy of the measurement of photogenerated current. If the input impedance is not negligible, then high measurement errors may occur. This unfavourable situation results in a low closed loop gain. If during a measurement cycle any of the impedances change this results in a change of the low closed loop gain that causes amplitude error. Either the detector has a low internal impedance or a high impedance detector is applied in high sensitivity measurements that requires high feedback impedance, this situation may occur. The impedance of the detector can be increased virtually by a factor of the open loop gain of the operational amplifier by using the bootstrapping technique, consequently, this error will appear only at a much higher sensitivity range i.e. at much higher feedback impedance. It restores the range-to-range ratio to its designed value for some further decades of the sensitivity range. The ultimate limit is the low-pass filter formed by the high value feedback resistance together with the inevitable stray capacitance. The signal - to - noise characteristic of the bootstrapped variation essentially did not change compared to the traditional solution.

\section{REFERENCES}

1. Makai, JP, Makai, T

Enhancement of the low level detection limit of radiometric quality photovoltaic and photoconductive detectors

Metrologia 42 (4), p. 266 (2005)

2. Rogalski, A, Adamiec, K. and Rutkowsi, J.

Narrow-gap semiconductor photodiodes

SPIE Press 2000

3. Deremiak, E.L. and Crowe, D.G.

Optical radiation detectors

John Wiley \& Sons 1984.

4. Lin, RM., Tang, SF., Lee, SC. and Kuan, CH.

Improvement of current leakage in the InAs photodetector by molecular beam epitaxy

Journal of Crystal Growth 227-228 p. 167-171 (2001)

5. Makai, JP. and Cromer, CL.

The Application of an Optical Biasing Method to Determine Temperature-dependent Nonlinearity of Photovoltaic Ge Detectors

Metrologia 30 (4), p. 335 (1993)

6. Campastro, OJ.

Revista Telegrafica Electronica 1984 p 832

7. Makai, JP. and Makai, JJ.

Current to voltage converter for linearity correction of low shunt resistance photovoltaic detectors Rev. Sci. Instruments 67 p 2381 (1996) 